\title{
Germanica
}

philosophique

\section{Die Freiheit eines Heiteren: Ernst Jüngers Marmorklippen}

Liberté et sérénité: Sur les falaises de marbre d'Ernst Jünger

Freedom and serenity: Ernst Jünger's On the Marble Cliffs

\section{Elisa Primavera-Lévy}

\section{OpenEdition}

\section{Journals}

Édition électronique

URL : http://journals.openedition.org/germanica/5419

DOI : 10.4000/germanica.5419

ISSN : 2107-0784

\section{Éditeur}

Université de Lille

\section{Édition imprimée}

Date de publication : 31 décembre 2018

Pagination : 111-126

ISBN : 978-2-913857-42-1

ISSN : 0984-2632

\section{Référence électronique}

Elisa Primavera-Lévy, „Die Freiheit eines Heiteren: Ernst Jüngers Marmorklippen“, Germanica [Online] 63 | 2018, Online erschienen am: 01 Januar 2021, abgerufen am 22 Januar 2021. URL: http:// journals.openedition.org/germanica/5419; DOI: https://doi.org/10.4000/germanica.5419 


\title{
Die Freiheit eines Heiteren: Ernst Jüngers Marmorklippen
}

\author{
Elisa PrIMAVERA-LÉVY \\ Berlin
}

\begin{abstract}
„Die Heiterkeit gehört zu den gewaltigen Waffen, über die der Mensch verfügt - er trägt sie als göttliche Rüstung, in der er selbst die Schrecken der Vernichtung zu bestehen vermag", schreibt Ernst Jünger 1938 in Das abenteuerliche Herz ${ }^{1}$. Diese Heiterkeit meint freilich nicht das schenkelklopfende selbstgenügsame Behagen oder das ungefährdete, sonnige Gemüt eines frohen Menschen. Als Amalgam aus stoisch-antiken und religiösen Traditionen sowie idealistischer, insbesondere Schiller'scher und Hegel'scher Bestimmungen zielt Jünger auf eine geistig reflektierte Form der Heiterkeit. Dieser liegt ein Erkennen zu Grunde, dass kein Entrinnen möglich ist, der Kampf keine Frucht bringt, dass nichts bleibt, als die gegebene Situation in Würde anzunehmen. Ein paradoxales Moment der Freiheit liegt in dem freiwilligen Sich-Fügen unter Zwang. Dahinter mag Gottvertrauen oder schlicht ein Vertrauen darauf stehen, dass es Größeres gibt als die eigene individuelle Existenz. Die idealistische Heiterkeit ist säkularisierte Heilssicherheit. Sie verspricht Zugang zu einem geistigen Raum, einem immateriellen panic room, in den man sich in höchster Anfechtung zurückziehen kann. Somit ist Heiterkeit,
\end{abstract}

1. - Ernst Jünger, Das abenteuerliche Herz. Figuren und Capriccios, Zweite Fassung, Hamburg, Hanseatische Verlagsanstalt, 1938, S. 126.

GERMANICA, 2018, LXIII, pp. 111 à 126. 
die auch am Diskurs des Erhabenen teilhat, das tertium comparationis von Heldentum und Gottvertrauen.

Diese knappen Charakterisierungen weisen bereits auf das Potential idealistisch gefasster Heiterkeit in Zeiten der Diktatur; für eine Haltung des inneren Widerstands bzw. der inneren Emigration kommt ihr ein besonderer Wert zu. Anhand Jüngers romanhafter Erzählung Auf den Marmorklippen (1939) sollen die philosophischen Zuflüsse sowie die poetologischen und politischen Konsequenzen seiner Heiterkeitsauffassung freigelegt werden.

\section{Heiterkeit als Aufklärung}

Nicht erst in Auf den Marmorklippen, sondern bereits in Das abenteuerliche Herz der ersten und zweiten Fassung $(1929,1938)$ häuft sich der Begriff der Heiterkeit; er bleibt fortan eine der zentralen Formeln im facettenreichen Jünger'schen Werk und mit der Bestimmung der eigenen Unverletzlichkeit verschränkt ${ }^{2}$. Drei Grundbedeutungen sind auszumachen: Heiterkeit wird zum einen als überraschend eintretender Aufklärungs-Effekt beschrieben. Zum anderen wird sie, wie eingangs zitiert, als Rüstung gefasst. Damit wird sie als innere Panzerung Voraussetzung und begleitendes Merkmal der mitleidsfreien, abgelösten Beobachtung.

Als reaktionärer Moderner vertritt Jünger ein gegenaufklärerisches Narrativ, das den kulturellen wie geistigen Niedergang in der Französischen Revolution verortet und den auf Vernunft, Wissenschaft, Humanismus und Fortschritt zielenden Aufklärungsbegriff bekämpft. Zugleich stilisiert er sich als Lichtbringer, der durch den vermeintlichen Verblendungszusammenhang der humanitären Zivilisation zur wahren Wirklichkeit leuchtet. Um die mannigfachen Zugänge zu den Räumen der tieferen Wirklichkeit zu kennzeichnen, bedient sich Jünger vornehmlich des Begriffs der „Stereoskopie“3. Im technisch gebräuchlichen Sinn bedeutet „Stereoskop“ ein sich durch die Kombination aus zwei zweidimensionalen Bildern ergebender räumlicher Tiefeneindruck. Jünger verwendet das Wort für eine synchrone Wahrnehmung aller Weltdinge, die sowohl die Oberflächendetails der materialen Gegebenheiten als auch die Einsicht in die unwandelbare und wunderbare, gleichsam ploti-

2. - Die Jünger-Forschung hat dies spätestens zum 100. Geburtstag Ernst Jüngers vermerkt; ein Werk der umfänglichen Jubiläumsprosa trug den Titel Magie der Heiterkeit, hg. v. Heimo Schwilk und Günter Figal, Stuttgart, Klett-Cotta, 1995.

3. - Die ersten Formulierungen des stereoskopischen Blicks finden sich bei Jünger in Das abenteuerliche Herz. Aufzeichnungen bei Tag und Nacht, Berlin, FrundsbergVerlag, 1929 (fortan zitiert als Das abenteuerliche Herz I) und in ,Sizilianischer Brief an den Mann im Mond“ in E. Jünger, Blätter und Steine, Hamburg, Hanseatische Verlagsanstalt, 1934, S. 110-124. 
nische Tiefenstruktur des Seins umfasst, in der alles mit allem verbunden ist. Stereoskopische Wahrnehmungen, ob sinnlicher oder geistiger Art, entstehen durch das sich Übereinanderschieben zweier Erscheinungen. Diesen eignet ein Angriffs-Moment der „Gleichgewichtsstörung“, des „Schwindels“, der „Erschütterung“, dem heiteres Entzücken folgt, wenn unvermutet in einem das „Andere“ hervortritt ${ }^{4}$. Dieses „Andere“ ist zugleich aber „Bestätigung“, also Erkennen des zeitlosen Mythos, der Urform, der Substanz oder Idee: ,Es gibt an dieser Tafel keine Speise, in der nicht ein Körnchen vom Gewürz der Ewigkeit enthalten ist" "5. Jünger hat die Erfahrung des momentanen Gleichgewichtverlusts, die sich in Heiterkeit auflöst, als ,Vexierbild“ formalisiert ${ }^{6}$. Im Staunen, das sich im Moment des Umschlags zeigt, hat Heiterkeit hier zur Komik eine natürliche Nähe. Deren Definitionen beinhalten häufig einen strukturellen Kipp-Moment, so etwa bei Odo Marquard, der als komisch bezeichnete, „was im offiziell Geltenden das Nichtige und im offiziell Nichtigen das Geltende sichtbar werden läßt" ${ }^{\text {"7 }}$. Stereoskopisch ist auch der Moment, wenn Tod und Leben plötzlich ihre Plätze wechseln, wie etwa im Krieg, wenn der Gegner überraschend aus dem wie toten Gelände auftaucht und die „mechanisch taktische Welt des Soldaten“ in einem ,plötzlichen Einsturz des Bewußtseins“ in die „dämonische des Kriegers“ verwandelt $^{8}$. Stereoskopische Erfahrungen führen durch das erheiternde Erstaunen zum Ansichtigwerden des Wunderbaren - zu den wirklicheren Schichten des Lebens und zur intuitiven Erkenntnis, dass dieses mehr ist als die von Jünger als schal-humanitär geächtete Oberfläche der Zivilisation. Die stereoskopische Sicht auf die Welt, die sich zwar als klare Wahrnehmung ausgibt, ist eine lebensphilosophisch unterfütterte Lenkung des Blicks. Ebenfalls dem Bereich der Stereoskopie zugeschlagen wird somit auch die Wahrnehmung der, ,geheime[n] doppelte[n] Buchführung"9, die dort, wo der Aufgeklärte nur sinnlose (Kriegs-) Verluste verbucht, einen Gewinn an einer entfernten Stelle ortet. Die stereoskopische Weltsicht als Lobpreis auf die den meisten unsichtbar bleibende Überfülle des Daseins, die Untergang, Neugeburt, Leid und Freude zu gleichen Teilen einschließt, ist Antidot gegen die rationalistische, antiheroische Moderne. Die Heiterkeit begleitet die Erkenntnis

4. - E. Jünger, Das abenteuerliche Herz I, a.a.O., S. 114, S. 205 sowie Das abenteuerliche Herz. Figuren und Capriccios, a.a.O., S. 34 (fortan zitiert als Das abenteuerliche Herz, II).

5. - E. Jünger, Das abenteuerliche Herz II, a.a.O., S. 34.

6. - Ebd., S. $136 \mathrm{f}$.

7. - Odo Marquart, „Exile der Heiterkeit“, in: Wolfgang Preisendanz, Rainer Warning (Hrsg.), Das Komische (Poetik und Hermeneutik 7), München, Wilhelm Fink Verlag, 1976, S. 133-151, hier S. 141.

8. - E. Jünger, Das abenteuerliche Herz I, a.a.O., S. 205.

9. - E. Jünger, Das abenteuerliche Herz II, a.a.O., S. 21. 
der anderen wunderbaren, ästhetischen und spirituellen Räume. Die Stereoskopie ist das (poetologische) Verfahren ihrer Herbeiführung.

\section{Heiterkeit als Rüstung}

Die Bewahrung der heiteren Gemütsruhe im Verderben und auf verlorenem Posten - ,eine Art von erlauchter Heiterkeit“10, wie es in Das abenteuerliche Herz heißt - ähnelt nicht von ungefähr der vergegenständlichenden „Kälte des zweiten Bewußtseins“ aus Jüngers bekanntem Essay Über den Schmerz (1934) ${ }^{11}$. Denn auch der Heitere kultiviert ein Bewusstsein, das unter Heranziehung einer als tiefer verstandenen Auslegung der Situation und der Dinge konsequent von allen schmerzlichen Oberflächenerscheinungen $\mathrm{zu}$ abstrahieren weiß. Neben Konnotationen eines würdigen, selbst am Rande der Auslöschung aufrechterhaltenen Selbstbesitzes entsteht so aber auch das Bild gänzlicher Empathielosigkeit, ja eines Indifferenten, der vor sich hinlächelt, während ringsum Verzweiflung um sich greift. Heiterkeit als Glaube an die eigentliche Unzerstörbarkeit der Substanz umschließt neben der spirituellen Trostfunktion auch die Voraussetzung der ästhetischen Untergangslust, die Jüngers Schriften durchsetzt. Tatsächlich verkörpert Jüngers Verwendung des Heiterkeitsbegriffs die andere, dunkle Seite der Heiterkeit - wenn man will, das Erbe der idealistischen, von körperlichen Dingen, Partikularitäten und damit auch konkret vom individuellen Leiden Einzelner abstrahierenden Philosophie. Heißt es doch charakteristisch bei Hegel im Hinblick auf die von den Modernen als kalt empfundene „Allgemeinheit“ der griechischen Götter: „,[D]er selige Frieden, der sich in ihrer Leiblichkeit abspiegelt, ist wesentlich ein Abstrahieren von Besonderem, ein Gleichgültigsein gegen Vergängliches, ein Aufgeben des Äußerlichen, ein nicht kummervolles und peinliches - doch ein Entsagen dem Irdischen und Flüchtigen, wie die geistige Heiterkeit tief über Tod, Grab, Verlust, Zeitlichkeit hinwegblickt"12. Hegels Vorlesungen zur Ästhetik (gehalten von 1817 bis 1829) diskutieren den Begriff der Heiterkeit im unmittelbaren Zusammenhang mit der Heldenproblematik. Die Heiterkeit fasst Hegel als wesentliches Attribut des Kunstideals. Die ideale Kunstgestalt verkörpert die perfekte Verbindung aus dem Allgemeinen und Individuellen und liegt im unbewegten Zustand, idealtypisch verkörpert in einer unbewegten Götterstatue, die „Unwandelbarkeit“ und „heitere Ruhe“ ausstrahlt ${ }^{13}$.

10. - Ebd., S. 130.

11. - „Über den Schmerz“, in: Ernst Jünger, Blätter und Steine, Hamburg, Hanseatische Verlagsanstalt, 1934, S. 157-216, hier: S. 206.

12. - G.W.F. Hegel, Vorlesungen über die Ästhetik II, Werke 14, Frankfurt a. M., Suhrkamp (suhrkamp taschenbuch wissenschaft 613), 1986, S. 68.

13. - Ders., Vorlesungen über die Ästhetik I, Werke 13, Frankfurt a. M., Suhrkamp 
Damit ist die Heiterkeit zugleich der stoischen Ataraxie verwandt, die als Unerschütterlichkeit des Gemüts, Seelenruhe und zustandshafte Lust umschrieben werden kann ${ }^{14}$. Zentral für Hegels Kunstideal ist aber wiederum eine Individualität, die die das Ideal störende Kollision durch die Handlung zur Harmonie führt und die Ruhe wiederherstellt. Dies bildet den Kern des Heiterkeitsbegriffs in der klassisch-idealistischen Theorie - eine Heiterkeit, die als geistige Widerständigkeit aufgefasst wird - in der Terminologie der zeitgenössischen Psychologie spräche man von Resilienz ${ }^{15}$.

Wir können in dieser Rücksicht die heitere Ruhe und Seligkeit, dieses Sichselbstgenügen in der eigenen Beschlossenheit und Befriedigung als den Grundzug des Ideals an die Spitze stellen. [...] Diese Kraft der Individualität, dieser Triumph der in sich konzentrierten konkreten Freiheit ist es, den wir besonders in den antiken Kunstwerken in der heiteren Ruhe ihrer Gestalten erkennen. Und dies ist nicht etwa bei kampfloser Befriedigung allein der Fall, sondern dann selbst, wenn ein tiefer Bruch das Subjekt in sich selbst wie dessen ganze Existenz zerrissen hat. Denn wenn die tragischen Heroen z.B. auch so dargestellt sind, daß sie dem Schicksale unterliegen, so zieht sich dennoch das Gemüt, in dem es sagt: Es ist so!, in das einfache Beisichsein zurück [...]. Der Mensch, vom Geschick unterjocht, kann sein Leben verlieren, die Freiheit nicht. Dies Beruhen auf sich ist es, welches im Schmerz selbst noch die Heiterkeit der Ruhe zu bewahren und erscheinen zu lassen vermag 16 .

Die Heiterkeit erscheint hier ungeschieden als sittliche und ästhetische Kategorie. Sie ist geistige Tugend. Die idealen Kunstgestalten macht sie trotz Schlägen unberührbar, lässt sie abstrahieren von den materiellen, dem Zwang unterworfenen, körperlichen Oberflächenbewegungen und partikularen Leidensvorkommnissen. Der heitere Mensch, der auch im Moment der höchsten Entzweiung, Anfechtung und Todesnähe bei sich ist, erlebt Freiheit. Heiterkeit ist eine Errungenschaft des Geistes, der sich transzendierend über die sinnliche Natur hinwegsetzt und nur so den Freiheitsanspruch lebbar macht. Ähnlich - wenngleich auch ohne explizite Verwendung des Begriffs der Heiterkeit - fasste Friedrich Schiller

(suhrkamp taschenbuch wissenschaft 613), 1986, S. 208.

14. - H. Reiner, „Ataraxie“, in: Joachim Ritter (Hrsg.), Historisches Wörterbuch der Philosophie, Bd. 1: A-C Basel, Schwabe \& Co. Verlag, 1971, Spalte 593.

15. - Vgl. etwa Robert Brooks, Sam Goldstein, Das Resilienz-Buch. Wie Eltern ihre Kinder fürs Leben stärken - das Geheimnis der inneren Widerstandskraft, Klett-Verlag, Stuttgart 2007; A. Gebauer, U. Kiehl-Dixon, „Das Nein zur eigenen Wahrnehmung ermöglichen - Umgang mit Extremsituationen durch Aufbau organisationaler Fähigkeiten“, Zeitschrift Organisationsentwicklung. Nr. 3, Düsseldorf 2009, S. 40-49.

16. - G.W.F. Hegel, Vorlesungen über die Ästhetik I, a.a.O., S. $208 \mathrm{f}$. 
zwei Jahrzehnte zuvor in seinem Aufsatz „Ueber das Erhabene“ (1801) die Möglichkeit der moralischen Freiheit. Nichts sei dem Menschen so unwürdig, als Gewalt zu erleiden, denn diese hebe ihn auf. Die Kultur setze den Menschen in Freiheit, sie gebe ihm sowohl Begriffe wie auch reelle Mittel, die Natur zu beherrschen. Dort aber, wo sich die Kräfte der Natur nicht mehr durch die physische Kultur beherrschen ließen, komme seine idealistische Anlage ihm zur Hilfe ,,[...] eine Gewalt, die er der That nach erleiden muß, dem Begriff nach zu vernichten. Eine Gewalt dem Begriffe nach vernichten, heißt aber nichts anders, als sich derselben freiwillig zu unterwerfen"17. Die Kultur, die den Menschen dazu ,geschickt macht“, heißt nach Schiller, die moralische. Nur sie setze ihn in den Stand vollendeter und nicht vom gnädigen Zufall abhängiger Freiheit.

Hegels Begrifflichkeit wird tendenziell ausschließlich als höhere geistige Heiterkeit aufgefasst, die sich als dialektisches "Ambivalenzphänomen"18 erst im Durchgang durch das negative Moment der Entzweiung, also durch „Tod, Grab, Verlust, Zeitlichkeit“ vollendet ${ }^{19}$. Heiterkeit wäre somit das siegreiche Gefühl der Seele, die den Kampf gekämpft hat, ,das Sinnliche und Endliche in sich ausgetilgt hat" und über Leiden triumphiert ${ }^{20}$. Tatsächlich haben aber sowohl die immanente Heiterkeit der Götter, die sich um nichts Menschliches kümmern, als auch die natürliche Heiterkeit, die nicht reflektiert ist, sondern als gegebene Disposition ausgewählten Individuen und göttergleichen schönen Seelen eigen ist, Teil an Hegels Ideal. So zeigen auch unvergeistigte Kunstgestalten der Genremalerei, wie z.B. die sorglos Trauben und Melonen verspeisenden Bettlerknaben auf einem Bild des barocken Malers Murillo heitere Ruhe und ,eine gänzliche Unbekümmertheit und Sorglosigkeit", also ihre Unabhängigkeit von ihrer misslichen materiellen Situation: „,Diese Kummerlosigkeit um das Äußere und die innere Freiheit im Äußeren ist es, welche der Begriff des Idealen erheischt. 21 “

Die natürliche, vom Bewusstsein ungetrübte Heiterkeit, die den göttergleichen, in ungesonderter Lebenskraft jenseits der Moral vernichtend wirkenden antiken Helden eignete, ist ein Wunschbild, das als Vorstellung weiterlebt. In Auf den Marmorklippen dekliniert Jünger anhand der Figuren des Kindes Erio, des Oberförsters und des

17. - Friedrich Schiller, „Ueber das Erhabene“, in: Schillers Werke. Nationalausgabe. Bd. 21 (1963), S. 38-54, hier S. 39.

18. - Harald Weinrich, Kleine Literaturgeschichte der Heiterkeit, München, C.H. Beck Verlag, S. 31.

19. - G.W.F. Hegel, Vorlesungen über die Ästhetik II, a.a.O., S. 68.

20. - Ders., Vorlesungen über die Ästhetik III, Werke 15, Frankfurt a. M., Suhrkamp (suhrkamp taschenbuch wissenschaft 613), 1986, S. 49.

21. - G.W.F. Hegel, Vorlesungen über die Ästhetik I, a.a.O., S. 224. 
Christenmönchs Pater Lampros drei Formen der Heiterkeit durch: als „Désinvolture“ - Jünger benützt seit der ersten Verwendung in dem Werk Das abenteuerliche Herz diesen französischen Ausdruck, um die gelassene und unwiderstehliche „Anmut“ oder „Unschuld der Macht“ zu bezeichnen; als ,furchtbare Jovialität“ der autochthonen Gewaltnatur und als höhere, idealistisch geprägte, leuchtende Heiterkeit des geistigen Menschen, der damit die „Sicherheit im Nichts“ erreicht ${ }^{22}$.

\section{Heitere Vorbereitung auf den Ernstfall: Auf den Marmorklippen}

Auf den Marmorklippen, die traumartig-allegorische Erzählung vom Untergang einer uralten Kultur durch die Kräfte des unversieglichen Bösen, schloss Jünger im Sommer 1939, noch kurz vor seinem zweiten Kriegseinsatz $a^{23}$. Laut vielfältiger Zeugnisse von Zeitgenossen im Inund Ausland wurde das schmale Bändchen unmittelbar nach Erscheinen als verschlüsselte Kampfansage an die nationalsozialistische Diktatur und als Widerstandsbuch verstanden ${ }^{24}$. Dolf Sternberger erinnerte sich 1980: „Es war wie ein Signal, das plötzlich aus der Düsternis aufschießt und die Gegend erhellt. Es bot Stärkung und wirkte als ein Mittel der Verständigung unter denen, die gegen Bedrohung oder Versuchung der Tyrannei sich festigten“25. Die erzählte Welt gleicht hinsichtlich Ort, Zeit und Geschehen einem Vexierbild, das teilweise aufgelöst werden kann. Als opak bleibendes Ganzes aus Chiffren der Zeitgeschichte, autobiographischen Elementen ${ }^{26}$, archaisch-mythischen und mittelalterlichen Versatzstücken im Stile Heinrich von Ofterdingens widersetzt es sich jedoch der einfachen Lesart eines Schlüsselromans des Dritten Reichs.

22. - Ernst Jünger, Auf den Marmorklippen [1939], Hamburg, Hanseatische Verlagsanstalt, 1941, S. 82.

23. - Jünger vermerkt am 10.9.1939 in seinem Tagebuch: „Merkwürdig übrigens, wie ich diese Arbeit ,zum Termin' beendete. Vielleicht gibt es Instanzen, die dafür sorgen, daß zu den Gerichten, wie sie die Zeit bereitet, ein jeder mit seinem Gewürz zur Stelle sei." E. Jünger, Strahlungen. Erster Teil, in: Ders., Werke. Bd. 2: Tagebücher, Stuttgart, Ernst Klett Verlag, 1962, S. 73.

24. - Vgl. etwa Günter Scholdt, „,Gescheitert an den Marmorklippen: Zur Kritik an Ernst Jüngers Widerstandsroman“, Zeitschrift für deutsche Philologie 98 (1979), S. 543-577.

25. - Dolf Sternberger, „Eine Muse konnte nicht schweigen“, in: Ders., Romane von gestern - heute gelesen. Bd. 3 (1933-1945), Frankfurt a. M., 1990, S. 199-208, hier: S. 199.

26. - Informierte Leser verglichen das Setting mit dem Überlingener „Weinbergshaus“, in dem Jünger und häufig auch sein Bruder Friedrich Georg bis 1939 wohnten. Sie wussten auch durch Jüngers Aufsätze und das abenteuerliche Herz um die magischen Vokal - und Sprachstudien. 
Im Mittelpunkt des kulminierenden Geschehens stehen der Ich-Erzähler und sein Bruder Otho, die sich nach einer kriegerischen Vergangenheit in die „Rautenklause“ zurückgezogen haben, um sich dort die ,Zeit absaugend“" mit Worten und Pflanzen und der in deren Erscheinung eingeschlossenen ewigen Ordnung $\mathrm{zu}$ beschäftigen. In diesen Studien leben die Brüder in einer üppigen, Bodensee, Rio de Janeiro, und ägäisches Meer vereinenden Phantasielandschaft ${ }^{27}$, am Fuße der Marmorklippen. Die Wissenschaft vom Überfluss sei die Theologie, heißt es in dem Werk Das abenteuerliche Herz in einer Überlegung zu Hesiods Diktum, dass die Götter den Menschen die Nahrung verbergen. Im zuversichtlichen Duktus enthüllen atmosphärisch dichte Naturbeschreibungen die Bausteine einer ,neue[n] Theologie" 28 , indem sie die Zugänge zum Überfluss, d.h. zum unzerstörbaren Lebensgrund freilegen.

Ihre Klause teilen die Brüder mit Erio, dem kleinen Söhnchen des Ich-Erzählers und dessen Wärterin, seiner Großmutter Lampusa, die hexenhafte Züge trägt. Von klein auf sucht Erio die Nähe der buntschillernden, aber hochgiftigen Lanzenottern, die aus den Klüften der Marmorklippen kommen, und bringt ihnen allabendlich ein „Kesselchen Milch“:

Und wie im Helltraum hörte ich den kleinen Erio lachen, als er zwischen ihnen auf dem gestampften Lehm des Küchen-Vorhofs stand. Die Tiere umspielten ihn halb aufgerichtet und wiegten über seinem Scheitel im schnellen Pendelschlage die schweren Dreiecksköpfe hin und her. Ich stand auf dem Altan und wagte meinen Erio nicht anzurufen, wie jemand, den man schlafend auf steilen Firsten wandeln sieht. Doch da erblickte ich die Alte vor der Felsen-Küche - Lampusa, die mit gekreuzten Armen lächelte, und es erfaßte mich das herrliche Gefühl der Sicherheit in flammender Gefahr ${ }^{29}$.

Die Art und Weise, in der Jünger das traumwandlerisch sichere Gebaren des Kindes beschreibt, erinnert stark an seine erste Beschreibung der Désinvolture in Das abenteuerliche Herz als ,göttergleich[e] Überlegenheit"،, als Macht, die nicht als Resultat eines übermächtigen Willens entsteht, sondern als „Wuchs und freie Gabe“. Kein Zweifel bestünde dort über Machtfragen, wo die Désinvolture noch intakt sei. Désinvolture, eine Tugend, dessen Äquivalent Jünger in der deutschen Sprache vermisst, ist „Ungeniertheit“, die aber nichts Unverschämtes an sich hat, da sie sich auf einen immanenten, von allen als selbstverständ-

27. - Vgl. Jüngers Tagebuch-Notizen zu den Modellen der Marmorklippen, Strahlungen. Erster Teil, a.a.O., S. 43.

28. - E. Jünger, Das abenteuerliche Herz II, a.a.O., S. 201.

29. - E. Jünger, Auf den Marmorklippen, a.a.O., S. 15. 
lich anerkannten Machtanspruch stützen kann. Der mit Désinvolture Gesegnete ist im vollen Gleichgewicht in Ausübung dessen, was ihm gefällt. Durch die Semantik der Balance und der gleichsam bewusstlosen, graziösen Bewegung schließt Jünger an Heinrich von Kleists Gedanken in Über das Marionettentheater an. Demjenigen, der die Macht als sein von den Göttern gegebenes Erbteil ohne Überlegung ausübe, drohe weder Neid noch Rebellion. Exemplifiziert wird dies am Beispiel eines Kindes, das an einer festlichen Tafel den ausgestellten, von allen brennend begehrten goldenen Apfel mit „,freier Hand“ ergreift, worauf sich aller Gäste eine tiefe Zustimmung bemächtigt. Jünger folgert: „Die Désinvolture als die unwiderstehliche Anmut der Macht ist eine besondere Form der Heiterkeit" $" 30$.

Eine verwandte Form der Désinvolture, wenngleich in vergröberter Ausrichtung, zeigt sich in der Figur des „Oberförsters“, einem Charismatiker der Macht, dessen sich ausbreitende Terrorherrschaft das brüderliche Idyll bedroht. Im Oberförster und seiner Gefolgschaft der „Lemuren“, Gewaltnaturen aus den als barbarisch gekennzeichneten Wäldern, erkannten zeitgenössische Leser Züge der Nazi-Spitzen - so insbesondere den passionierten Jäger Hermann Göring - sowie ihrer sozial deklassierten Anhänger ${ }^{31}$. Der Oberförster, dem Souveränität und „fürchterlich[e] Jovialität ${ }^{32 “}$ aus den Augen blitzen, verkörpert die ungesonderte Lebenskraft eines Machtmenschen, der nicht am Bewusstsein kränkelt, sondern durch seine rohen, im Vollbesitz seiner Kräfte verübten Untaten Faszination verbreitet. „Grandezza“ und „angeboren[e] Désinvolture“ lassen ihn die Gewalt wie einen „guten, alten Jagdrock“ tragen, „,er stets bequemer wird, je öfter er sich mit

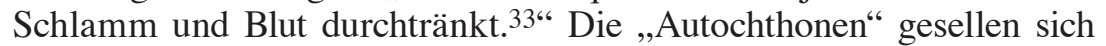
ihm zu ,wie die Schlange zu den offenen Feuern kriecht. Sie sind die alten Kenner der Macht und sehen eine neue Stunde tagen, die Tyrannis wieder aufzurichten, die seit Anbeginn in ihren Herzen lebt" 34 Zeitgenossen mochten darin Jüngers kühle Verachtung für die nationalsozialistische Plebiskratie ausmachen, die Herrschaft des Pöbels, für die er die NSDAP schon als Publizist der nationalrevolutionären Bewegung geschmäht hatte.

Tatsächlich erscheinen viele Aussagen - wie etwa über die Sprache, die sich mit Worten durchsetzt, , die sonst dem Ungeziefer galten, das ausgerottet, vertilgt und ausgeräuchert werden soll“" oder über die bei „Nacht

30. - E. Jünger, Das abenteuerliche Herz II, a.a.O., S. 125.

31. - D. Sternberger, „Eine Muse...“, a.a.O., S. 203 f.

32. - E. Jünger, Auf den Marmorklippen, a.a.O., S.29.

33. - Ebd., S. 107.

34. - Ebd., S. 33. 


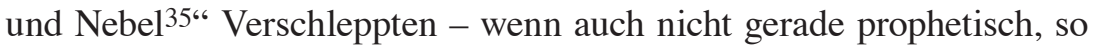
doch wortmächtig gebannt und dadurch von „befreiender Wirkung“36. (Bei Erscheinen des Buchs 1939 waren Regimegegner bereits nächtlich massenhaft in „Schutzhaft“ festgesetzt worden). Berühmt geworden ist Jüngers alptraumhafte, an die Capriccios des Abenteuerlichen Herzens erinnernde Beschreibung der im Wald verborgenen "Schinderhütte“ von Köppelsbleek, eines archetypischen Angstorts der Marter und absoluten Auslieferung, in dem spätere Kommentatoren ein Proto-KZ erblickten ${ }^{37}$. Der Oberförster steht am Kopf der „Mauretanier“, eines weitverzweigten Ordens von faschistischen Gruppierungen, die die Macht, unter Ausschluss moralischer Fragen, rein technisch behandeln. In dieser Luft, „die von Grund auf böse war“, hielten sich auch die Brüder in einer nihilistisch orientierungslosen Phase ihres früheren Lebens auf, fasziniert vom psychopathischen Übermut derjenigen, die „gleich Adlern über dumpfen Leiden kreisen“:

Einmal, als wir mit dem Capitano tranken, blickte er in den betauten Kelch wie in ein Glas, in dem vergangene Zeiten sich erschließen, und er meinte träumend: „Kein Glas Sekt war köstlicher als jenes, das man uns an die Maschine reichte in der Nacht, da wir Sagunt zu Asche brannten." Und wir dachten: Lieber noch mit diesem stürzen, als mit jenen leben, die die Furcht im Staub zu kriechen zwingt ${ }^{38}$.

Diesem Weg des empathielosen Tätertums wird unter Verweis auf die den Brüdern versagte Gabe, ,auf das Leiden der Schwachen und Namenlosen herabzusehen, wie man vom Senatoren-Sitze in die Arena blickt" ${ }^{\text {"39 }}$, eine eindeutige Absage erteilt, die häufig als autobiographisches Eingeständnis einer moralischen Umkehr Jüngers und Verurteilung seines früheren politischen Engagements als Publizist gelesen wird ${ }^{40}$.

Die Brüder entschließen sich gegen den Ausweg der physischen Gewalt im aktiven Kampf gegen den Oberförster und seine „Lemuren“. Dies hat auch seinen Grund darin, dass sie letztere nicht als Gegner ansehen, gegen die sie, wie im zurückliegenden Feldzug von „Alta Plana“, ritterlich kämpfen könnten, sondern als „niedere Bosheit“, als gleichsam dämonisch besessene Untermenschen. Hier greift eine kriegerische Ideal-Ethik, der zufolge der Feind zwar um jeden Preis

35. - Ebd., S. 50 und 54.

36. - D. Sternberger, „Eine Muse...“, a.a.O., S. 200.

37. - Eduard Lachmann, ,Die Sprache der Marmorklippen“, Wirkendes Wort 2 (1953/54), S. 91-101, hier S. 96.

38. - E. Jünger, Auf den Marmorklippen, a.a.O., S. 32.

39. - Ebd., S. 62.

40. - So z.B. Helmut Kiesel, „Ernst Jüngers ,Marmor-Klippen‘: ,Renommier'- und ,Problembuch“ der 12 Jahre“, IASL 14 (1989), S. 126-164, hier: S. 137. 
zu vernichten, aber als Kämpfer zu ehren und nicht menschlich zu verachten sei. Bezeichnend ist der Satz: „So billigten wir dem LemurenVolke nicht Willensfreiheit zu“41. Auch die Pläne des Fürsten Sunmyra und des Mauretaniers Braquemart, den Oberförster durch ein Attentat $\mathrm{zu}$ vernichten, unterstützen die Brüder nicht. Stattdessen widmen sie sich, angeleitet durch das Beispiel des Christenmönchs Lampros, ihren Pflanzenstudien und dem Widerstand durch „reine Geistesmacht" ${ }^{\text {“42. }}$. Die geistige Heiterkeit der stereoskopischen Betrachtung, durch die sich „die Lust zu leben und die Lust zu sterben“ in ihnen eint, bestimmt das Handeln. Sie wird zu einer Ethik des Nichteingreifens, aber auch des Nichtausweichens. In der Dramaturgie der Erzählung alternieren die Beschreibungen der Verheerungen mit kontemplativen Passagen, welche die Freiheit raunend beschwören: als Reiche, die jenseits der Zerstörung liegen, als „Sicherheit im Nichts“, die man in der „Stunde der Vernichtung" heiter betreten werde ${ }^{43}$. Zur Hälfte der Erzählung, im 15. Kapitel, wird den Brüdern in plotinisch-stereoskopischer Schau von den Klippen auf das weit unter ihnen liegende Land die für das Werk zentrale, in meditativen Sätzen entfaltete Einsicht zuteil, dass den Mächten der Zerstörung Schranken gesetzt sind und die eigentliche Substanz unzerstörbar bleibt:

Vom höchsten Grat der Klippen, der im Mittag blendend und fernhin leuchtete, sahen wir lange auf das Land, und unsere Blicke suchten sein Heil in jeder Falte, in jedem Raine zu erspähen. Dann fiel es uns wie Schuppen von den Augen, und wir begriffen es, so wie die Dinge in den Gedichten leben, im Glanze seiner Unzerstörbarkeit.

Und freudig erfaßte uns das Wissen, daß die Vernichtung in den Elementen nicht Heimstatt findet, und daß ihr Trug sich auf der Oberfläche gleich Nebelbildern kräuselt, die der Sonne nicht widerstehn. Und wir erahnten: wenn wir in jenen Zellen lebten, die unzerstörbar sind, dann würden wir aus jeder Phase der Vernichtung wie durch offene Tore aus einem Festgemach in immer strahlendere gehen ${ }^{44}$.

Dieser angesichts drohender Vernichtung heiteren Fassung, ja Heilserwartung liegt, wie so oft bei Jünger, die Prämisse einer unhintergehbaren - zwischen Schicksal und Biologie changierenden - Notwendigkeit der Entwicklungen zugrunde. Von inneren Zersetzungsprozessen geschwächt hatte die Marina den barbarischen Einflüssen Haus und Hof geöffnet und war reif zum Untergang: „Die Festen wankten und die Zeit war für die Fürchterlichen reif.

41. - E. Jünger, Auf den Marmorklippen, a.a.O., S. 85.

42. - Ebd., S. 75.

43. - Ebd., S. $82 \mathrm{f}$.

44. - Ebd., S. 77. 
Die Menschen-Ordnung gleicht dem Kosmos darin, daß sie von Zeit zu Zeiten, um sich von neuem zu gebären, ins Feuer tauchen muß ${ }^{\prime \prime 4}$. An dieser und ähnlichen Passagen zeigt sich, dass in Jüngers Heiterkeitsbegriff nicht nur idealistische Bestimmungen wirken, welche die Freiheit im Moment der Katastrophe beschwören. Es fließen in ihm auch Vorstellungen religiösen Schicksals- und Jenseitsglaubens mit organischen Kultur-Modellen zusammen.

Kritik an den Marmorklippen, ein weiterer notorischer Testfall der Jünger-Rezeption, blieb nicht aus. Moniert wurde der auf Erhabenheit zielende Edelkitsch und pretiöse Stil eines „musealen Neuklassizismus"46 mit semantischen Archaismen wie "Tannicht" oder „Glaste“, die manieristische Syntax mit „Relativsatzschleppe“ und die gehäuften Satzanfänge mit „Auch“, „Dann“ und „So“47 sowie die gezwungen wirkende Rhythmisierung der Prosa, auf die Jünger, wie er in den Tagebüchern vermerkte, viel Arbeit verwendete ${ }^{48}$. Auch die Entwirklichung der physischen Gewalt durch die märchenhaft-archaische Kulisse einerseits und die Beschwörung der unzerstörbaren Zellen andererseits erschien, insbesondere mit Bekanntwerden der Naziverbrechen, zunehmend problematisch. Zu Schluss der Schilderung der Schinderstätte Köppelsbleek heißt es provozierend über die „Höchsten“, die diesem entwürdigenden Anblick gewachsen bleiben, weil sie den tiefsten Schrecken als Trugbild erkennen: „Sie wissen, daß alle diese Bilder ja nur in unserem Herzen leben, und schreiten als durch vorgestellte Spiegelungen durch sie in stolze Siegestore ein “49. Im Zusammenhang mit der kritisierten Derealisierung steht schließlich der schon mit Erscheinen des Kriegsbuchs In Stahlgewittern (1922) vorgebrachte Vorwurf der ästhetisch überhöhten Darstellung der Vernichtung 50 .

Den Untergang der Marina erlebt der Erzähler vom schmerzlosen, erhöhten Ort der Marmorklippen in einer nahezu paralysierten „Traumes-Starre“ und obendrein geräuschlos. Es zeichnet sich eine Anthropologie des Mitleids ab: Nur über das Ohr sind Menschen moralische Tiere, über das Auge ästhetische:

45. - Ebd., S. 62.

46. - Rezension von S. (Redaktionskürzel) zu Aufden Marmorklippen, Hochland: Monatsschrift für alle Gebiete des Wissens, der Literatur und Kunst 37, 1 (1939-40), S. 244-245.

47. - E. Lachmann, „Die Sprache der Marmorklippen“, a.a.O., S. 91-101.

48. - E. Jünger, Strahlungen. Erster Teil, a.a.O., S. 30; 60.

49. - E. Jünger, Marmorklippen, a.a.O., S. 97.

50. - Wolfgang Kaempfer, Ernst Jünger, Stuttgart, Metzler Verlag, 1981. (= Sammlung Metzler. Realien zur Literatur, Band 201), S. 72 ff. 
Als ob der Raum ganz luftleer wäre, drang nicht ein Laut herauf; das Schauspiel dehnte sich in fürchterlicher Stille aus. Ich hörte dort unten nicht die Kinder weinen und die Mütter klagen, auch nicht das Kampfgeschrei der Sippenbünde und das Brüllen des Viehes, das in den Ställen stand. Von allen Schrecken der Vernichtung stieg zu den Marmor-Klippen einzig der goldene Schimmer. So flammen ferne Welten zur Lust der Augen in der Schönheit des Untergangs auf ${ }^{51}$.

An solchen Tod, Untergang und farbenprächtige Schönheit verknüpfenden Szenen zeigt sich die „fatale Affinität“ mit den Vernichtungsphantasmen, Weltenbrand- und Untergangsmythen, die selbstverständlich keine originär nationalsozialistischen Hervorbringungen waren, aber vom Regime politisch funktionalisiert werden konnten ${ }^{52}$. Auf den ideologischen „Widerschein des Nazismus“53 als Ästhetisierung von Gewalt und Vernichtung in Jüngers Bildgebung wiesen vor allem diejenigen Rezipienten hin, die die Erzählung nach 1945 nicht als Widerstandsparabel gelten lassen wollten. Sentenzartig bekannt geworden ist das in einem Brief vom Dezember 1945 gefällte Urteil Thomas Manns:

Es [die Marmorklippen] ist das Renommierbuch der 12 Jahre und sein Autor zweifellos ein begabter Mann, der ein viel zu gutes Deutsch schrieb für Hitler-Deutschland. Er ist aber ein Wegbereiter und eiskalter Genüßling des Barbarismus und hat noch jetzt, unter der Besetzung, offen erklärt, es sei lächerlich, zu glauben, daß sein Buch mit irgendwelcher Kritik am nationalsozialistischen Regime zu tun habe ${ }^{54}$.

Nach Kriegsende war Jünger den Besatzungsmächten verdächtig. $\mathrm{Ob}$ er sich einem Spruchkammerverfahren bzw. dem Ausfüllen eines Entnazifizierungsfragebogens entzogen hat, ist nicht eindeutig zu klären. Bis 1949 hatte er Publikationsverbot, dem er sich schließlich durch einen Umzug von der britischen in die französische Besatzungszone, nach Ravensburg, entziehen konnte. Seit Dezember 1933 hatte Jünger mit seiner Familie fern der Hauptstadt in der deutschen Provinz gelebt, in Goslar, Überlingen und Kirchhorst. Er tat dies auch, um sich der Aufmerksamkeit von Seiten der neuen Machthaber zu entziehen, die ihm 1933 ein Reichstagsmandat antrugen und ihn in die gleichgeschaltete Deutsche Akademie der Dichtung wählten ${ }^{55}$. Beide Vorschläge

51. - E. Jünger, Auf den Marmorklippen, a.a.O., S. 143.

52. - H. Kiesel, ,Ernst Jüngers ,Marmor-Klippen’..., a.a.O., S. 158.

53. - Saul Friedländer, Kitsch und Tod.Der Widerschein des Nazismus, München, Hanser Verlag, 1986, S. 21 ff.

54. - T. Mann, Briefe 1937-1947, hg. v. Erika Mann, Berlin und Weimar, AufbauVerlag, 1965, S. 495.

55. - Nennenswert ist ein Brief an den Völkischen Beobachter, in dem sich Jünger 
lehnte Jünger ab und lebte bis zu seiner Einberufung, so wie in den Marmorklippen vorgezeichnet, durch „reine Geistesmacht", d.h. durch gärtnerische, schriftstellerische und ordnende (in seinem Fall Käfer) Arbeit in „innerer Emigration“. Dennoch lässt sich, wie nach Kriegsende häufig geschehen, darauf hinweisen, dass ein Autor vorrangig durch seine Bücher wirkt. „Der Dichter Jünger hat wie kaum ein anderer vor ihm die Möglichkeiten der deutschen Sprache ausgeschöpft, um die deutsche Jugend gegen den Schauder vor kommenden Kriegen zu immunisieren“, schrieb Anfang 1946 Eugen Gürster-Steinhausen ${ }^{56}$. Neuauflagen von In Stahlgewittern und Der Kampf als inneres Erlebnis, die von den Herrschenden als positive, wehrstärkende Vorbereitung der nationalsozialistischen Jugend eingestuft wurden, verhinderte Jünger nicht ${ }^{57}$.

Jüngers Selbstaussagen hinsichtlich der Marmorklippen als Widerstandsbuch variieren mit den Zeitumständen. Die Erzählung wollte er 1939, womöglich auch aus Selbstschutz, nicht nur allegorisch verstanden wissen, sondern als zeitloses, wiederkehrendes, also mythisches Bild eines Geschehens ${ }^{58}$. Im Tagebuch wird auch die Reaktion seines Bruders Friedrich Georg überliefert, der im Gespräch über die „politischen Auspizien“ bemerkte: „Das verbieten sie dir entweder in den ersten vierzehn Tagen oder nie“ 59 . Dass die Erzählung nicht der Zensur anheimfiel, ist erstaunlich. Dies mag Hitlers sentimentaler Verehrung für den hoch dekorierten Kriegsschriftsteller oder auch taktischen Überlegungen geschuldet gewesen sein; ein Verbot wäre dem Eingeständnis der Machthaber gleichgekommen, dass sie die Charakterisierung als „Waldgelichter“, „Lemuren“ und mordende Oberförster trifft. Noch 1946 hielt Jünger in seinem Tagebuch fest, das Buch sei keine „Tendenzschrift" und die geschilderte Lage kein spezifisch deutsches Problem, sondern ließe sich als Bild eines in den Untergang gehenden, von nihilistischen Zeitströmungen zersetzten Gemeinwesens auf verschiedenste geschichtliche Zeitpunkte anwenden ${ }^{60}$. In seinen späten Tagebüchern Siebzig Verweht wählte Jünger dagegen bevorzugt

1934 ausdrücklich gegen einen unautorisierten Abdruck aus Das abenteuerliche Herz verwehrt. Der Brief endet mit „Mein Bestreben läuft nicht darauf hinaus, in der Presse möglichst oft genannt zu werden, sondern darauf, daß über die Art meiner politischen Substanz auch nicht die Spur einer Unklarheit entsteht.“ Zitiert nach: Joseph Wulf, Literatur und Dichtung im Dritten Reich, Gütersloh, Siegbert Mohn Verlag, 1963, S. 11.

56. - Eugen Gürster-Steinhausen, „Ernst Jünger - der Prophet des deutschen Nationalismus“, Die Neue Rundschau, H. 2 1/1946 Januar 1946, S. 238-245.

57. - Wolfgang Brekle, „,Das Unbehagen Ernst Jüngers an der Nazi-Herrschaft“, Weimarer Beiträge 40 (1994), S. 335-350, hier: S. 345 f.

58. - E. Jünger, Strahlungen. Erster Teil, a.a.O., S. 29.

59. - Ebd., S. 66.

60. - Ernst Jünger, Strahlungen. Zweiter Teil, in: Werke. Bd. 3: Tagebücher III, Stuttgart, Ernst Klett Verlag, 1962, S. 615. 
die Figur des prophetischen Sehers, der die Dinge in der Art des zweiten Gesichts, als „Vorbrand“ zu Papier gebracht habe, wozu ihm selbst die Ereignisse erst den „Schlüssel“ gegeben hätten ${ }^{61}$. Nach dem ihm 1982 verliehenen Goethe-Preis und links-intellektuellen Anfeindungen bemühte sich Jünger verstärkt darum, seine integre Haltung während der zwölf Jahre nationalsozialistischer Herrschaft und der Pariser Besatzungszeit ins rechte Licht zu rücken. Dabei spielt das Schicksal der Marmorklippen eine ausnehmende Rolle und Jünger nimmt nun eine klare Einordnung derselben als Akt des Widerstands vor. So zitiert er in Siebzig Verweht III ausführlich aus der Korrespondenz mit Werner Best (1982/83), Heydrich-Stellvertreter und einem der letzten Überlebenden der obersten NSDAP-Führung. Dieser bestätigte ihm, dass Reichsleiter Bormann wegen Jüngers „, antinationalsozialistischem Schlüsselroman“ eine politische Beurteilung einholen ließ ${ }^{62}$. In einem Brief von 1983 an eine Vertraute des Kreises der „Weißen Rose“ erfragte Jünger, ob die Geschwister Scholl sein Buch häufig verschenkt hätten. Ihn interessiert auch, ob die Gruppe die Marmorklippen nicht nur gelesen, wovon Jünger selbstverständlich ausgeht, sondern auch laut vorgelesen hätten $^{63}$. Dies alles ist Teil der Tagebücher, die Jünger mit Bedacht zur Veröffentlichung auswählte und mit seiner Korrespondenz kompilierte.

Fazit

Für Ernst Jünger, der seine literarische Karriere als Schilderer von Kriegshandlungen begonnen hatte und bis in die späten 1920er Jahre als Kämpfer mit der Feder für die nationalrevolutionäre Sache wirkte, bot die in der zweiten Werkhälfte hervortretende Formel der Heiterkeit zweierlei: Sie erlaubte ihm, die kriegerische Semantik mit einem zwischen Philosophie und Weltweisheit changierenden Idiom zu ersetzen, ohne die Glaubenssätze einer heldischen Weltanschauung aufzugeben: den Opfermut, die mit dem Tod rechnende Pflichterfüllung, die geforderte Tugend des heroischen Realismus im Angesicht des sicheren Untergangs, die bedingungslose Unterordnung und konsequente Abwertung des einzelnen Menschenlebens unter eine wie auch immer bestimmte Sache (Freiheit, Tiefe, Erhabenheit, Notwendigkeit) oder eine unbestimmt bleibende Sache im Glauben an eine höhere Substanz. Darüber hinaus verbirgt sich in der Darstellung der Heiterkeit als kontemplative Naturbeobachtung, in der sich die plato-

61. - Ernst Jünger, Siebzig Verweht III, in Sämtliche Werke. Bd. 20, Stuttgart, Klett-Cotta Verlag, 1993, S. 53.

62. - Ebd., S. 231-239.

63. - Ebd., S. 284. Die Praxis des Vorlesens war offenbar gängig. Vgl. Alfred Anderschs Zeugnis in „Achtzig und Jünger“, Merkur 29 (1975), S. 242: „Diese hoch-romantische und symbolistische Erzählung war für uns alle die Parabel der Notwendigkeit von Widerstand. Wir lasen sie, lasen sie uns gegenseitig vor, begriffen, antizipierten in ihr den Untergang von Hitlers Reich.“ 
nisch gefügten Urformen als Ort des Unzerstörbaren enthüllen, ein fruchtbares poetologisches Verfahren, das er bis zu seinen allerletzten Publikationen beibehielt. Schließlich gelang mit der Heiterkeit und dem Verweis auf einen unverfügbaren, sicheren Innenraum sowie mit der Darstellung von Mönchen als Propagatoren der Heiterkeitslehre ${ }^{64}$, die Anbindung, wenn auch nicht an eine orthodox-christliche, jedoch an eine religiös-mystische Diskurssphäre. Die sogenannte christlich-humanitäre Wende Jüngers nach dem Zweiten Weltkrieg, in der er seinem früheren heroischen Nihilismus abschwor, zeigt sich nicht zuletzt in den Heiterkeitspostulaten und den stetigen Verweisen auf einen unhintergehbaren Schicksalsgrund, angesichts dessen die Empfehlungen der Religion traditionell auf die Ergebung in den göttlichen Ratschluss verwiesen 65 .

64. - Pater Lampros in Auf den Marmorklippen; Pater Foelix in Heliopolis. Rückblick auf eine Stadt, Tübingen, Heliopolis Verlag, 1949.

65. - Vgl. hierzu Gottfried Benn, Doppelleben. Zwei Selbstdarstellungen. Stuttgart, Klett-Cotta Verlag, 1984, S.183: „Eine neue große Woge von Frömmigkeit geht über den Erdteil. Döblin, einst großer Avantgardist und Franz Biberkopf vom Alexanderplatz, wurde streng katholisch und verkündet Ora et labora, Toynbee ist christlich, Eliot ebenso, Jünger gibt sich christlich-humanistisch - alles Rückgriffe, schöne Haltung, aber Stilentspannung, Konformismus. Ich versage mir diese Rückgriffe, ich kann auch diese Frage nur artistisch sehen.“ 